\title{
Efeitos da cinesioterapia na osteoartrite de joelho em idosos: revisão sistemótica
}

\section{Effects of kinesiotherapy on knee osteoarthritis in elderly: a systematic review}

\author{
Matheus Santos Gomes Jorgel; Caroline Zanin²; Bruna Knob33; Julia Dal Pozzo Comin ${ }^{4}$ \\ Igor Moreira ${ }^{5}$; Lia Mara Wibelinger ${ }^{6}$ \\ 1 Curso de Fisioterapia, Especialização em Fisioterapia Traumato-ortopédica, Programa de Pós-Graduação em Envelhecimento \\ Humano, Faculdade de Educação Física e Fisioterapia, Bolsista Prosuc/Capes. Universidade de Passo Fundo - UPF. Soledade, RS - \\ Brasil. \\ 2 Curso de Fisioterapia, Faculdade de Educação Física e Fisioterapia, Universidade de Passo Fundo - UPF. São Jorge, RS - Brasil. \\ 3 Curso de Fisioterapia, Faculdade de Educação Física e Fisioterapia, Universidade de Passo Fundo - UPF. Chapada, RS - Brasil. \\ 4 Curso de Fisioterapia, Faculdade de Educação Física e Fisioterapia, Universidade de Passo Fundo - UPF. Nova Prata, RS - Brasil. \\ 5 Curso de Fisioterapia, Faculdade de Educação Física e Fisioterapia, Universidade de Passo Fundo - UPF. Carazinho, RS - Brasil. \\ 6 Gerontologia Biomédica, Pontifícia Universidade Católica do Rio Grande do Sul - PUCRS. Curso de Fisioterapia e Programa de Pós- \\ Graduação em Envelhecimento Humano, Faculdade de Educação Física e Fisioterapia, Universidade de Passo Fundo - UPF. Passo \\ Fundo, RS - Brasil.
}

Endereço para Correspondência: Matheus Santos Gomes Jorge

Rua Rio de Janeiro, $n^{\circ}$ 797, Bairro Ipiranga

99300-000 - Soledade, RS [Brasil]

matheussgjorge@gmail.com

\begin{abstract}
Resumo
Introdução: a osteoartrite é uma doença articular inflamatória e degenerativa que acomete principalmente os joelhos, sobretudo em idosos. Objetivo: verificar os efeitos da cinesioterapia na osteoartrite de joelho em idosos. Métodos: realizou-se uma revisão sistemática nas bases de dados SciELO, LILACS, Cochrane Library e MEDLINE, onde cruzaram-se os descritores "Osteoartrite", "Fisioterapia", "Exercício", "Terapia por exercício" e "Idoso", nas línguas portuguesa, inglesa e espanhola, considerando-se os artigos originais publicados entre 2010 e 2016. Resultados: encontraram-se 594 artigos. Inicialmente 62 artigos potencialmente relevantes foram selecionados para o estudo. Após uma análise qualitativa criteriosa, selecionaram-se 06 artigos completos que preencheram os critérios de inclusão exigidos, cujos mesmos foram analisados por meio da Escala PEDro. Conclusão: a cinesioterapia (aquecimento, alongamento dos músculos posteriores da coxa e fortalecimento dos músculos anteriores da coxa), com frequência de duas vezes por semana, em um período de seis a 12 semanas, parece ser benéfica para melhorar o desempenho muscular, funcionalidade, diminuir a dor e rigidez em idosos com osteoartrite de joelho, sem a necessidade de recursos adicionais.
\end{abstract}

Descritores: Osteoartrite; Fisioterapia; Exercício; Terapia por exercício; Idoso.

\section{Abstract}

Introduction: Osteoarthritis is an inflammatory and degenerative joint disease that mainly affects the knees, especially in the elderly. Objective: To verify the effects of kinesiotherapy in knee osteoarthritis in the elderly. Methods: a systematic review was carried out in the databases SciELO, LILACS, Cochrane Library and MEDLINE, where they crossed the descriptors "Osteoarthritis", "Physiotherapy", "Exercise", "Exercise therapy" and "Elderly", in Portuguese, English and Spanish. considering the original articles published between 2010 and 2016. Results: 594 articles were found. Initially 62 potentially relevant articles were selected for the study. After a careful qualitative analysis, we selected six complete articles that met the inclusion criteria required, which were analyzed using the PEDro Scale. Conclusion: kinesiotherapy (warming, stretching of the posterior thigh muscles and strengthening of the anterior thigh muscles), often twice a week, over a period of six to 12 weeks, seems to be beneficial to improve muscle performance, functionality, decrease pain and stiffness in the elderly with knee osteoarthritis, without need for additional resources.

Keywords: Osteoarthritis; Physical therapy specialty; Exercise; Exercise therapy; Aged. 


\section{Introdução}

A osteoartrite (OA) é uma doença articular inflamatória degenerativa resultante das respostas mediadas por condrócitos e sinoviócitos. Sua etiologia é multifatorial que envolve mudanças no alinhamento oateomioarticular e causando, consequentemente, instabilidade articular. Além disso, os níveis de soro e de citocinas inflamatórias sinoviais são mais elevados nos seus acometidos em comparação aos indivíduos não acometidos pela doença ${ }^{1-3}$.

No Brasil, a OA atinge $16 \%$ da população. Trata-se da forma mais comum de artrite e uma das dez doenças reumáticas mais incapacitantes e prevalentes na população idosa, acometendo preferencialmente o sexo feminino ${ }^{4-7}$.

Embora possa acometer quaisquer regiões do corpo, atinge preferencialmente as articulações que suportam grandes quantidades de peso e que requerem frequente utilização, como é o caso dos joelhos ${ }^{2}$. Os achados clínicos incluem o aumento do volume articular, em virtude de uma sinovite causada por um derrame ou espessamento sinovial, dor ao repouso, rigidez matinal, incapacidade, fraqueza muscular e perda de função, impactando a funcionalidade, o processo da marcha, as atividades de vida diária e a qualidade de vida destes indivíduos ${ }^{8-11}$.

Estudos recentes demonstram que o exercício físico e a educação do indivíduo, associados às outras abordagens não farmacológicas, são os tratamentos mais indicados para a $\mathrm{OA}^{12}$. A cinesioterapia convencional pode ser uma intervenção benéfica no trofismo muscular, na força e na resistência muscular, na amplitude de movimento articular e na funcionalidade de portadores da doença ${ }^{13}$.

Desta forma, o presente estudo buscou revisar na literatura fontes que documentem os efeitos da cinesioterapia na OA de joelho em idosos.

\section{Métodos}

Este é um estudo do tipo revisão sistemática, na qual foram consultados manualmente ar- tigos indexados nas bases de dados eletrônicos SciELO, LILACS, Cochrane Library e MEDLINE, no período de janeiro de 2010 a maio de 2016.

A estratégia de busca dos artigos partiu do descritor primário "Osteoartrite" em cruzamento com descritores secundários como "Fisioterapia", "Exercício", "Terapia por exercício" e "Idoso". Também, realizou-se uma busca com as variações destas palavras nas línguas inglesa e espanhola, sendo elas, respectivamente, "Osteoarthritis/ Osteoartritis", "Physical therapy specialty/ Fisioterapia", "Exercise/ Ejercicio", "Exercise therapy/ Terapia por ejercicio" e "Aged/ Anciano". Todos os descritores estavam de acordo com os Descritores em Ciência da Saúde (DeCS). O período de realização do estudo foi entre os meses de junho a julho de 2016.

Os critérios de inclusão adotados neste estudo foram:

- Estudos do tipo ensaio clínico randomizado;

- Estudos que caracterizassem o tema "cinesioterapia na OA de joelho em idosos";

- Estudos que foram publicados livremente na íntegra na base de dados supracitadas.

- Mediante isto, os critérios de exclusão adotados neste estudo foram:

- Estudos com abordagem medicamentosa ou cirúrgica;

- Estudos que não envolvessem idosos;

- Estudos com envolvimento de OA em qualquer outra articulação, exceto o joelho.

$\mathrm{Na}$ fase inicial três revisores independentes identificaram os artigos nas bases de dados e leram seus títulos e resumos, para selecionar aqueles que atendessem aos critérios de elegibilidade. Foram retidos para uma análise do texto na íntegra somente os artigos em que pelo menos dois revisores acreditassem ser potencialmente relevantes. Posteriormente, outros dois revisores independentes leram os artigos pré-selecionados na íntegra e identificaram aqueles que se encaixaram dentro dos critérios propostos pelo presente estudo. Em caso de divergência, um terceiro revisor seria requisita- 
do para fazer a análise do artigo em questão e determinar sua inclusão ou exclusão. Assim, a figura 1 apresenta a estratégia de busca e seleção dos artigos.

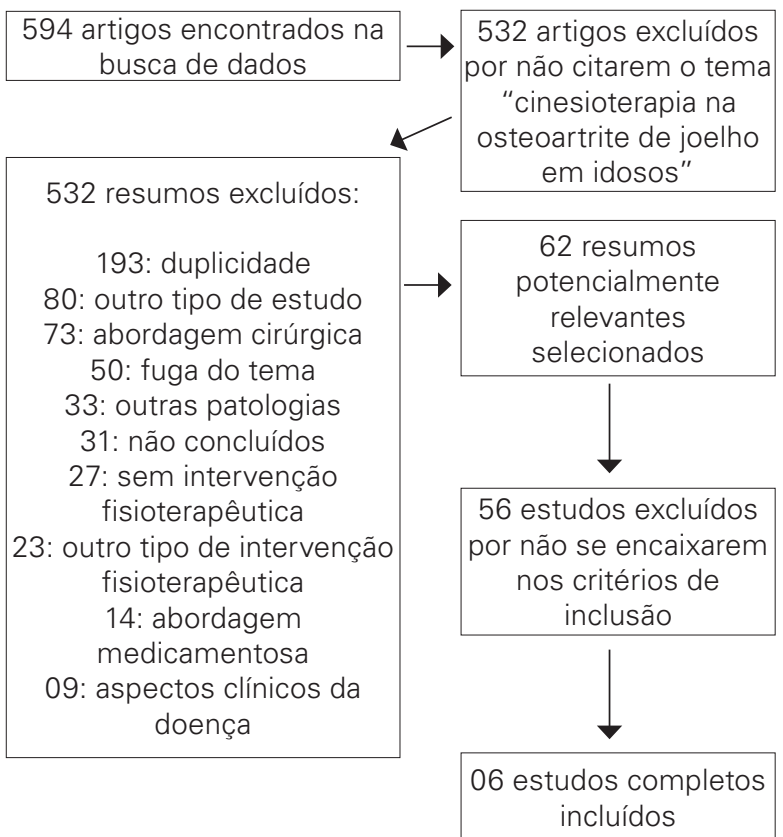

Figura 1: Fluxograma de estratégia de seleção dos artigos

Fonte: elaboração própria.

Inicialmente, foram identificados 594 artigos nas bases de dados, dos quais apenas 62 artigos foram considerados potencialmente relevantes para o estudo e foram pré-retidos. Após uma análise qualitativa de forma criteriosa dos textos na íntegra, selecionaram-se, por fim, 06 artigos completos que preencheram os critérios de inclusão exigidos e permitiriam a fundamentação teórica da presente pesquisa.

A análise da qualidade metodológica dos estudos foi realizada utilizando a Escala PEDro, uma escala específica para revisões sistemáticas de ensaios clínicos randomizados da área da Fisioterapia. Trata-se de uma escala de 11 critérios que incluem: (1) critérios de elegibilidade, (2) aleatoriedade entre grupos, (3) distribuição cega dos sujeitos, (4) semelhança entre grupos na analise inicial, (5) cegamento dos sujeitos, (6) cegamento dos terapeutas, (7) cegamento dos avaliadores, (8) 85\% de aproveitamento da amos- tra em um desfecho chave, (9) mesmo tempo de avaliação e análise dos dados para os grupos interdependente de ser intervenção ou controle, (10) comparações estatística inter-grupos em pelo menos um desfecho chave, e (11) presença de medidas de precisão e variabilidade. $\mathrm{O}$ escore da escala varia de 0 a 10 pontos, sendo que o primeiro item não é contabilizado. Quanto maior a pontuação melhor a qualidade do estudo. A Escala PEDro foi traduzida para a língua portuguesa em 2009 e ajustada para o Português brasileiro em 2010.

No parágrafo acima foram inseridas informações a respeito da Escala PEDro utilizada nesse estudo.

\section{Resultodos}

A Tabela 1 apresenta a caracterização das amostras dos estudos incluídos nesta revisão sistemática.

Dentre os estudos selecionados, o tamanho amostral variou de 33 a 183 indivíduos com OA, com médias de idade que variou de 60,1 anos a 67 anos. A maioria das amostras era do gênero feminino (576 mulheres e 97 homens). As articulações acometidas eram uni ou bilaterais em todos os estudos e os graus de OA variaram entre I (grau mais leve) ou IV (grau mais grave).

A Tabela 2 apresenta os instrumentos avaliativos e os protocolos interventivos utilizados, bem como os desfechos encontrados nos estudos incluídos nesta revisão sistemática.

Os trabalhos incluídos demonstram que a cinesioterapia parece benéfica no tratamento fisioterapêutico da OA de joelho em idosos. Embora 04 estudos utilizassem de uma abordagem "plus" no tratamento cinesioterapêutico, tais como a estimulação elétrica neuromuscular, a orientação sobre formas de estabilização do joelho ou a adição de exercícios de agilidade e propriocepção, tais medidas não se sobressaiam em relação ao tratamento cinesioterapêutico convencional, cujo mesmo demonstrou efeitos benéficos principalmente sobre a dor, a funcionalidade, a 
Tabela l: Caracterização das amostras dos estudos recrutados sobre os efeitos da cinesioterapia na osteoartrite de joelho em idosos

\begin{tabular}{c|c|c|c|c|c}
\hline Referência & Número & Idade & Gênero & Acometimento & $\begin{array}{c}\text { Grau de } \\
\text { osteoartrite }\end{array}$ \\
\hline $\begin{array}{c}\text { Teixeira, Piva e Fitzgerald, } \\
2011^{14}\end{array}$ & 183 & $\pm 64,4$ anos. & $\begin{array}{r}122 \text { mulheres } \\
61 \text { homens }\end{array}$ & Uni ou bilateral & I, II, III e IV \\
\hline $\begin{array}{c}\text { Sayers, Gibson e Cook, } \\
2011^{15}\end{array}$ & 33 & $\pm 67,0$ anos; & $\begin{array}{r}25 \text { mulheres } \\
8 \text { homens }\end{array}$ & Uni ou bilateral & I, II, III e IV \\
\hline $\begin{array}{c}\text { Imoto, Peccin e Trevisani, } \\
2012^{16}\end{array}$ & 100 & $\pm 60,1$ anos & $\begin{array}{r}92 \text { mulheres } \\
8 \text { homens }\end{array}$ & Uni ou bilateral & II, III ou IV \\
\hline Oliveira et al., 2012 & 100 & $\pm 60,1$ anos & $\begin{array}{r}92 \text { mulheres } \\
6 \text { homens }\end{array}$ & Uni ou bilateral & II, III ou IV \\
\hline Knoop et al., 2013 & 159 & $\pm 61,9$ anos & $\begin{array}{r}159 \text { mulheres } \\
0 \text { homens }\end{array}$ & Uni ou bilateral & I, II, III e IV \\
\hline Imoto et al., 2013 & 100 & $\pm 61,0$ anos & $\begin{array}{r}86 \text { mulheres } \\
14 \text { homens }\end{array}$ & Uni ou bilateral & II, III, IV \\
\hline
\end{tabular}

Fonte: elaboração própria.

rigidez, as atividades de vida diária e a qualidade de vida dos idosos com OA de joelho.

Adicionalmente, de acordo com a Escala PEDro, a qualidade metodológica dos estudos variou de 07 a 09 pontos.

\section{Discussão}

Os estudos encontrados sugerem que há interesse na comunidade científica sobre o tema proposto nesta revisão, visto que em um período de 06 anos há 06 estudos que contemplem os efeitos da cinesioterapia na OA de acordo com os critérios exigidos.

Atualmente, estima-se que a população acima dos 60 anos seja em torno de 19,6 milhões de habitantes (10,2\% da população total) e projeções futuras indicam que, em 2020, o Brasil será o sexto país no mundo em número de idosos, ultrapassando a casa dos 30 milhões de pesso$\mathrm{as}^{20}$. Com o aumento de indivíduos idosos, cresce também a ocorrência do número de doenças crônicas associadas ao envelhecimento, como é o caso da OA uma doença que afeta as articulações comprometendo a cartilagem hialina e o osso subcondral ${ }^{21,22}$.

Trata-se de uma doença comum entre as mulheres idosas e sua prevalência aumen- ta com a idade. A dor tende a exacerbar com a imobilidade prolongada, sendo que indivíduos sintomáticos apresentam pior qualidade de vida, funcionalidade e desempenho físico se comparados com os portadores assintomáti$\cos$ da doença ${ }^{23-26}$. A fraqueza da musculatura de membros inferiores, sobretudo do quadríceps femoral, está intimamente ligada a OA de joelho ${ }^{27}$. Por esta razão todos os estudos ${ }^{14-19}$ abordaram em seus protocolos exercícios de fortalecimento para os membros inferiores, especialmente do músculo quadríceps femoral, apresentando resultados positivos sobre as variáveis analisadas.

O WOMAC (Western Ontario and McMaster Osteoarthritis Index) é tido como o questionário mais adequado para avaliação das limitações físicas, cujo mesmo aborda a dor, a rigidez articular e a funcionalidade de indivíduos com $\mathrm{OA}^{28}$. Nesta revisão, cinco dos seis estudos recrutados utilizaram este questionário em sua avaliação visto sua aplicabilidade e especificidade para a $\mathrm{OA}^{14,15,17-19}$.

O tratamento farmacológico parece não promover resultados plenos sobre a funcionalidade destes indivíduos, sendo necessário o encorajamento de outras abordagens terapêuticas, como é o caso da cinesioterapia ${ }^{26}$. O exercício físico tem um possível efeito anti-inflamatório 
Tabela 2: Instrumentos avaliativos, protocolos interventivos e desfechos dos estudos sobre os efeitos da cinesioterapia na osteoartrite de joelho em idosos

\begin{tabular}{|c|c|c|c|}
\hline Referência & Instrumentos avaliativos e protocolos interventivos & Desfechos & $\begin{array}{c}\text { Análise da qualidade } \\
\text { metodológica pela } \\
\text { Escala PEDro }\end{array}$ \\
\hline $\begin{array}{l}\text { Teixeira, Piva } \\
\text { e Fitzgerald } \\
(2011)^{14}\end{array}$ & $\begin{array}{c}\text {-Avaliação: WOMAC, LEFS, ADLS; } \\
\text {-Intervenção: } \\
\text { G1 (n=84): cinesioterapia (alongamento de isquiostibiais, } \\
\text { fortalecimento muscular de quadríceps femoral, amplitude de } \\
\text { movimento articular e exercícios aeróbicos); } \\
\text { G2 (n=75): cinesioterapia (mesmo protocolo de G1)+ } \\
\text { exercícios de agilidade e propriocepção; } \\
\text {-TI: } 12 \text { sessões, } 2 x / \text { semana, } 06 \text { semanas. }\end{array}$ & $\begin{array}{l}0 \text { protocolo proposto para o } \mathrm{G} 2 \\
\text { não demonstrou superioridade } \\
\text { sobre o } \mathrm{G} 1 \text { no desempenho das } \\
\text { tarefas funcionais. }\end{array}$ & 07 \\
\hline $\begin{array}{l}\text { Sayers, Gibson e } \\
\text { Cook }(2011)^{15}\end{array}$ & $\begin{array}{c}\text {-Avaliação: WOMAC, TC400m, Escala de equilíbrio de Berg, } \\
\text { TUG, Teste de 1RM. } \\
\text {-Intervenção: } \\
\text { G1 (n=12): cinesioterapia (alongamento de isquiostibiais, } \\
\text { aquecimento, fortalecimento de quadríceps femoral em alta } \\
\text { velocidade); } \\
\text { G2 (n=10): cinesioterapia (alongamento de isquiostibiais, } \\
\text { aquecimento, fortalecimento de quadríceps femoral em baixa } \\
\text { velocidade); } \\
\text { G3 (n=11): controle (alongamento e ciclismo). } \\
\text {-Tl: } 36 \text { sessões, 3x/semana, } 12 \text { semanas. }\end{array}$ & $\begin{array}{l}0 \text { protocolo proposto para o G1 } \\
\text { foi mais efetivo no desempenho } \\
\text { muscular e parece ser mais } \\
\text { resistente e seguro. }\end{array}$ & 06 \\
\hline $\begin{array}{l}\text { Imoto, Peccin e } \\
\text { Trevisani }(2012)^{16}\end{array}$ & $\begin{array}{c}\text {-Avaliação: TUG, EVA, SF-36. } \\
\text {-Intervenção: } \\
\text { G1 (n=50): cinesioterapia (aquecimento, alongamento } \\
\text { muscular de ísquiostibiais, fortalecimento muscular de } \\
\text { quadríceps). } \\
\text { G2 (n=50): orientações domiciliares. } \\
\text {-TI: } 16 \text { sessões, } 2 x / \text { semana, } 08 \text { semanas. }\end{array}$ & $\begin{array}{l}\text { O protocolo proposto para o G1 } \\
\text { foi efetivo na melhora da dor, da } \\
\text { funcionalidade e da qualidade } \\
\text { de vida. }\end{array}$ & 06 \\
\hline $\begin{array}{l}\text { Oliveira et al. } \\
(2012)^{17}\end{array}$ & $\begin{array}{c}\text {-Avaliação: TUG, WOMAC, Índice de Lequesne. } \\
\text {-Intervenção: } \\
\text { G1 (n=50): cinesioterapia (aquecimento, alongamento } \\
\text { muscular de ísquiostibiais, fortalecimento muscular de } \\
\text { quadríceps). } \\
\text { G2 (n=50): orientações domiciliares. } \\
\text {-TI: } 16 \text { sessões, } 2 x / \text { semana, } 08 \text { semanas. }\end{array}$ & $\begin{array}{l}0 \text { protocolo proposto para o G1 } \\
\text { foi efetivo na melhora da dor, da } \\
\text { funcionalidade e da rigidez. }\end{array}$ & 08 \\
\hline $\begin{array}{c}\text { Knoop et al. } \\
(2013)^{18}\end{array}$ & $\begin{array}{l}\text {-Avaliação: WOMAC, EVA, percepção do indivíduo, avaliação } \\
\text { da estabilidade do joelho. } \\
\text {-Intervenção: } \\
\text { G1 (n=80): cinesioterapia (estabilização articular, fortaleci- } \\
\text { mento muscular, atividades de vida diária) + orientações sobre } \\
\text { a estabilização do joelho; } \\
\text { G2 (n=79): cinesioterapia (mesmo protocolo de G1). } \\
\text {-Tl: } 24 \text { sessões, } 2 x / \text { semana, } 12 \text { semanas. }\end{array}$ & $\begin{array}{l}\text { Ambos os grupos apresentaram } \\
\text { redução da dor e limitação das } \\
\text { atividades. Contudo, o protocolo } \\
\text { proposto para o G1 não } \\
\text { demonstrou superioridade sobre } \\
\text { o G2 nas variáveis analisadas. }\end{array}$ & 08 \\
\hline $\begin{array}{l}\text { Imoto et al. } \\
(2013)^{19}\end{array}$ & $\begin{array}{c}\text {-Avaliação: TUG, WOMAC, pontos de dor. } \\
\text {-Intervenção: } \\
\text { G1 (n=50): EENM + cinesioterapia (aquecimento, alongamento } \\
\text { de ísquiostibiais, fortalecimento de quadríceps femoral). } \\
\text { G2 (n= 50): cinesioterapia (mesmo protocolo de G1). } \\
\text {-Tl: } 16 \text { sessões, } 2 x / \text { semana, } 08 \text { semanas. }\end{array}$ & $\begin{array}{l}\text { Ambos os grupos melhoraram a } \\
\text { dor, a funcionalidade e a rigidez. } \\
\text { Contudo, o protocolo proposto } \\
\text { para o G1 não demonstrou } \\
\text { superioridade sobre o G2 nas } \\
\text { variáveis analisadas. }\end{array}$ & 08 \\
\hline
\end{tabular}

Legenda: ADLS: Activities of Daily Living Scale; EENM: estimulação elétrica neuromuscular; EVA: escala visual analógica; G1: grupo 1; G2: grupo 2; G3: grupo 3; HADS: Hospital Anxiety and Depression Scale; LEFS: Lower Extremity Function Scale; RM: repetição máxima; SF-36: Short Form Health Survey-36; TI: tempo de intervenção: TC: teste de caminhada; TUG: Timed Up and Go; WOMAC: Western Ontario and McMaster Osteoarthritis Index. Fonte: elaboração própria. 
em doenças reumáticas, o que pode atenuar a inflamação sistêmica e reduzir a ingestão medicamentosa ${ }^{29}$. Isso se deve ao fato de que a contração muscular induz às miocinas, cujas mesmas podem estar envolvidas em mecanismos que promovam efeitos benéficos para a saúde de indivíduos com doenças crônicas. A longo prazo, estes efeitos podem ser atribuídos à resposta anti-inflamatória provocada por um ataque agudo de exercício, que é parcialmente mediado pela Interleucina 6 derivada de músculo ${ }^{30-32}$.

Desta forma, a cinesioterapia busca aliviar a dor, melhorar a força muscular, a mobilidade, a flexibilidade, o equilíbrio postural e, consequentemente, promover a qualidade de vida e reduzir o risco de morbidade e mortalidade de indivíduos com $\mathrm{OA}^{2,7,26}$. Fatores estes que levaram à concepção do tema proposto por esta revisão sistemática.

O programa de exercícios para o joelho com OA busca melhora do desempenho da capacidade aeróbica, da força muscular do quadríceps femoral e da funcionalidade de membros inferiores, sendo indicados de modo supervisionado e pelo menos três vezes por semana ${ }^{27}$. O que vai de encontro a maioria dos estudos recrutados, visto que duas sessões semanais de cinesioterapia, em um período de seis a doze semanas, parece ser o suficiente para apresentar resultados satisfatórios nos desfechos analisa$\operatorname{dos}^{14,16-19}$.

Embora os exercícios de força muscular, apresentem bons resultados em relação a um grupo controle, os exercícios resistidos de alta intensidade não apresentam boa qualidade de evidências para o tratamento da OA de joelho. A funcionalidade parece ser a prioridade no tratamento de aumento da força muscular ${ }^{33}$. Um dos estudos apresentados demonstrou que os exercícios de fortalecimento em alta intensidade foram mais eficazes do que os de baixa intensidade. A justificativa para tal foi de que as atividades funcionais dependessem de vigorosas forças para serem executadas e que a abordagem de alta intensidade seria a mais resistente e segura ${ }^{15}$.

\section{Conclusão}

De acordo com esta revisão sistemática, observou-se que a cinesioterapia (aquecimento, alongamento dos músculos posteriores da coxa e fortalecimento dos músculos anteriores da coxa) realizada com frequência de duas vezes por semana, em um período de seis a 12 semanas, parece ser benéfica para melhorar o desempenho muscular, a funcionalidade, diminuir a dor e a rigidez em idosos com OA de joelho, sem a necessidade de recursos adicionais.

\section{Referências}

1. Sohn DH, Sokolove J, Sharpe O, Erhart JC, Chandra $\mathrm{PE}$, Lahey LJ, et al. Plasma proteins present in osteoarthritic synovial fluid can stimulate cytokine production via Toll-like receptor 4. Arthritis Res. Ther. 2012;14(1):7.

2. Duarte VS, Santos ML, Rodrigues KA, Ramires JB, Arêas GPT, Borges GF. Exercícios físicos e osteoartrose: uma revisão sistemática. Fisioter. Mov. 2013;26(1):193-202.

3. Coelho CF, Leal-Junior EC, Biasotto-Gonzalez DA, Bley AS, Carvalho PT, Politti F, et al. Effectiveness of phototherapy incorporated into an exercise program for osteoarthritis of the knee: study protocol for a randomized controlled trial. Trials. 2014;15(1):221.

4. McDonough CM, Jette AM. The contribution of osteoarthritis to functional limitations and disability. Clin. Geriatr. Med. 2010;26(3):387-99.

5. Bijlsma JW, Berenbaum F, Lafeber FP. Osteoarthritis: an update with relevance for clinical practice. Lancet. 2011;377(9783):2115-26.

6. Guermazi A, Niu J, Hayashi D, Roemer FW, Englund M, Neogi T, et al. Prevalence of abnormalities in knees detected by MRI in adults without knee osteoarthritis: population based observational study (Framingham Osteoarthritis Study). BMJ. 2012;345:e5339.

7. Cunha-Miranda L, Faustino A, Alves C, Vicente V, Barbosa S. Avaliação da magnitude da desvantagem da osteoartrite na vida das pessoas: estudo MOVES. Rev. Bras. Reumatol. 2015;55(1):22-30. 
8. Akyol Y, Durmus D, Alayli G, Tander B, Bek Y, Canturk F, et al. Does short-wave diathermy increase the effectiveness of isokinetic exercise on pain, function, knee muscle strength, quality of life, and depression in the patients with knee osteoarthritis? A randomized controlled clinical study. Eur J Phys Rehabil Med. 2010;46(3):325-36.

9. Rezend MU, Campos GC. Is osteoarthritis a mechanical or inflammatory disease? Rev. Bras. Ortop. 2013;48(6):471-474.

10. DiBonaventura MC, Gupta S, McDonald M, Sadosky A. Evaluating the health and economic impact of osteoarthritis pain in the workforce: results from the National Health and Wellness Survey. BMC Musculoskelet Disord. 2011;12(1):83.

11. Fernandes WC, Machado A, Borella C, Carpes FP. Influência da velocidade da marcha sobre a pressão plantar em sujeitos com osteoartrite unilateral de joelho. Rev. Bras. Reumatol. 2014;54(6):441-5.

12. Roos EM, Juhl CB. Osteoarthritis 2012 year in review: rehabilitation and outcomes. Osteoarthr. Cartil. 2012;20(1):1477-83.

13. Tok F, Aydemir K, Peker F, Safaz I, Kaynatan MAT, Ozgu A. The effects of electrical stimulation combined with continuous passive motion versus isometric exercise on symptoms, functional capacity, quality of life and balance in knee osteoarthritis: randomized clinical trial. Rheumatol. Int. 2011;31(1):177-81.

14. Teixeira PE, Piva SR, Fitzgerald GK. Effects of impairment-based exercise on performance of specific self-reported functional tasks in individuals with knee osteoarthritis. Phys Ther. 2011;91(12):1752-65.

15. Sayers SP, Gibson K, Cook CR. Effect of high-speed power training on muscle performance, function, and pain in older adults with knee osteoarthritis: a pilot investigation. Arthritis Care Res (Hoboken). 2012;64(1):46-53.

16. Imoto AM, Peccin MS, Trevisani VFM. Exercícios de fortalecimento de quadríceps são efetivos na melhora da dor, função e qualidade de vida de pacientes com osteoartrite do joelho. Acta Ortop. Bras. 2012;20(3):174-9.

17. Oliveira AMI, Peccin MS, Silva KNG, Teixeira LEPP, Trevisani VFM. Impacto dos exercícios na capacidade funcional e dor em pacientes com osteoartrite de joelhos: ensaio clínico randomizado. Rev. Bras. Reumatol. 2012;52(6):876-82.
18. Knoop J, Dekker J, Leeden M, Esch M, Thorstensson CA, Gerritsen M, et al. Knee joint stabilization therapy in patients with osteoarthritis of the knee: a randomized, controlled trial. Osteoarthr. Cartil. 2013;21(8):1025-34.

19. Imoto AM, Peccin S, Silva KNG, Teixeira LEPP, Abrahão MI, Trevisani VFM. Effects of neuromuscular electrical stimulation combined with exercises versus an exercise program on the pain and the function in patients with knee osteoarthritis: a randomized controlled trial. Biomed Res Int. 2013;2013:272018.

20. Tannure MC, Alves M, Sena RR, Chianca TCM. Perfil epidemiológico da população idosa de Belo Horizonte, MG, Brasil. Rev. Bras. Enferm. 2010;63(5):817-22

21. Wibelinger LM, Vidmar MF, Silveira MM, Presta SA, Batista JS. Caracterização e condições de saúde dos idosos do município de Passo Fundo, no Rio Grande do Sul. Rev. Bras. Ciênc. Saúde. 2011;9(29):31-8.

22. Ferreira AH, Godoy PBG, Oliveira NRC, Diniz RAS, Diniz REAS, Padovani RC, et al. Investigação da ansiedade, depressão e qualidade de vida em pacientes portadores de osteoartrite no joelho: um estudo comparativo. Rev. Bras. Reumatol. 2015;55(5):434-8.

23. Muraki S, Akune T, Oka H, En-yo Y, Yoshida M, Saika A, et al. Association of radiographic and symptomatic knee osteoarthritis with health related quality of life in a population based cohort study in Japan: the ROAD study. Osteoarthr Cartil. 2010;18(9):1227-34.

24. Kim IJ, Kim HA, Seo YI, Jung YO, Canção YW, Jeong JY, et al. Prevalence of knee pain and its influence on quality of life and physical function in the Korean elderly population: a community based crosssectional study. J. Korean Med. Sci. 2011;26(9):1140-6.

25. Imene KB, Zeineb S, Rim M, Rahma S, Leila M, Hajer RK. Epidemiology and clinical profile of knee osteoarthrosis in the elderly. Tunis Med. 2014;92(5):335-40.

26. Santos JPM, Andraus RAC, Oliveira Deise AAP, Fernandes MTP, Frâncica MC, Frederico RCP, et al . Análise da funcionalidade de idosos com osteoartrite. Fisioter. Pesqui. 2015;22(2):161-8.

27. Juhl C, Christensen R, Roos EM, Zhang W, Lund H. Impact of exercise type and dose on pain and disability in knee osteoarthritis: a systematic review and meta regression analysis of randomized controlled trials. Arthritis Rheumatol. 2014;66(3):622-36. 
28. Metsavaht L, Leporace G, Sposito MMM, Riberto M, Batista LA. Qual o melhor questionário para avaliar os aspectos físicos de pacientes com osteoartrite no joelho na população brasileira. Rev. Bras. Ortop. 2011;46(3):256-61.

29. Gualano B, Pinto ALS, Perondi MB, Roschel $\mathrm{H}$, Sallum AME, Hayashi APT, et al. Efeitos terapêuticos do treinamento físico em pacientes com doenças reumatológicas pediátricas. Rev. Bras. Reumatol. 2011;51(5):490-6.

30. Petersen AMW, Pedersen BK. The anti-inflammatory effect of exercise. J Appl Physiol. 2005;98(4):11541162 .
31. Pedersen BK. The anti-inflammatory effect of exercise: its role in diabetes and cardiovascular disease control. Essays Biochem. 2006;42:105-117.

32. Petersen AMW, Pedersen BK. The role of IL-6 in mediating the anti-inflammatory effects of exercise. J. Physiol. Pharmacol. 2006;57(suppl. 10):43-51.

33. Zacharias A, Green RA, Semciw AI, Kingsley MI, Pizzari T. Efficacy of rehabilitation programs for improving muscle strength in people with hip or knee osteoarthritis: a systematic review with metaanalysis. Osteoarthr. Cartil. 2014;22(11):1752-73. 